\title{
Revisão com síntese qualitativa sobre as experiências de meninos e meninas que
}

\section{praticam bullying na escola}

\author{
Review with quality synthesis about the experiences of boys and girls who are bullies at school \\ Revisión con síntesis cualitativa sobre las experiencias de los niños y niñas acosadores en la escuela
}

Recebido: 12/01/2022 | Revisado: 19/01/2022 | Aceito: 20/01/2022 | Publicado: 22/01/2022

\author{
Manuella Cana Brasil Feijó \\ ORCID: https://orcid.org/0000-0002-8338-3793 \\ Pontifícia Universidade Católica de Campinas, Brasil \\ E-mail: manuella.cbf@ puccampinas.edu.br \\ Victoria Maria Ribeiro Lembo \\ ORCID: https://orcid.org/0000-0003-3052-9047 \\ Pontifícia Universidade Católica de Campinas, Brasil \\ E-mail: vickmrlembo3@gmail.com \\ Karen Yukari Kimura \\ ORCID: https://orcid.org/0000-0001-9335-4104 \\ Pontifícia Universidade Católica de Campinas, Brasil \\ E-mail: karen.yukari.kyk@gmail.com \\ André Luiz Monezi Andrade \\ ORCID: https://orcid.org/0000-0003-0111-8935 \\ Pontifícia Universidade Católica de Campinas, Brasil \\ E-mail: andre.andrade@ @uc-campinas.edu.br \\ Julliane Messias Cordeiro Sampaio \\ ORCID: https://orcid.org/0000-0003-2781-9051 \\ Centro Universitário de Brasília, Brasi \\ E-mail: julliane.sampaio@ ceub.edu.br \\ Wanderlei Abadio de Oliveira \\ ORCID: http://orcid.org/0000-0002-3146-8197 \\ Pontifícia Universidade Católica de Campinas, Brasil \\ E-mail:wanderleio@hotmail.com
}

\begin{abstract}
Resumo
Esse estudo focalizou a questão da prática do bullying escolar e as diferenças entre meninos e meninas. Objetivou-se sintetizar e interpretar estudos primários qualitativos sobre a prática de bullying escolar. Metodologicamente, consultou-se as bases de dados PsycINFO, SCOPUS, Web of Science, SciELO e o periódico International Journal of Bullying Prevention. Seguiu-se a seguinte questão norteadora: quais as evidências científicas qualitativas sobre a as experiências de meninos e meninas que praticam bullying na escola? Foram incluídos na revisão seis artigos. Verificou-se que os meninos praticam mais o bullying do tipo físico e as meninas utilizam técnicas indiretas para agredir colegas. Os agressores, em geral, apresentam as seguintes características: alto senso de superioridade; desejo de controle; busca de poder e status; obtenção de prazer; agressividade como constituinte de sua imagem; consciência da prática da intimidação. Em uma nova síntese foi apresentada uma crítica reflexiva sobre os achados revisados. Embora o número de referências incluídas na revisão seja limitado, essa síntese qualitativa é relevante na medida em que lança luz sobre as experiências de meninas e meninas que praticam bullying e essas evidências poderão nortear ações de intervenção antibullying.
\end{abstract}

Palavras-chave: Violência; Saúde escolar; Bullying; Metasíntese; Revisão.

\begin{abstract}
This study focused on the issue of the practice of school bullying and the differences between boys and girls. We aim to synthesize and interpret qualitative primary studies on the practice of school bullying. Methodologically, we consulted the PsycINFO, SCOPUS, Web of Science, SciELO databases and the periodic International Journal of Bullying Prevention. The guiding question are: what is the qualitative scientific evidence on the experiences of boys and girls who practice bullying at school? Six articles were included in the review. It was found that boy's practice more physical type bullying and girls use indirect techniques to attack colleagues. Aggressors, in general, have the following characteristics: high sense of superiority; desire for control; seeking power and status; obtaining pleasure; aggressiveness as a constituent of its image; awareness of the practice of bullying. In a further synthesis a reflective critique of the reviewed findings was presented. Although the number of references included in the review is limited, this meta-synthesis is relevant as it sheds light on the experiences of girls and girls who practice bullying, and this evidence may guide antibullying intervention actions.
\end{abstract}

Keywords: Violence; School health; Bullying; Meta-synthesis; Revision. 


\begin{abstract}
Resumen
Este estudio se centró en el tema de la práctica del acoso escolar y las diferencias entre chicos y chicas. Nuestro objetivo fue sintetizar e interpretar estudios cualitativos primarios sobre la práctica del acoso escolar. Metodológicamente, se consultaron las bases de datos PsycINFO, SCOPUS, Web of Science y SciELO y International Journal of Bullying Prevention. Se siguió la pregunta guía: ¿cuál es la evidencia científica cualitativa sobre las experiencias de niños y niñas que practican el bullying en la escuela? Se incluyeron seis artículos en la revisión. Se comprobó que los chicos son más propensos al acoso físico y las chicas utilizan técnicas indirectas para atacar a sus compañeros. Los agresores, en general, tienen las siguientes características: alto sentido de superioridad; deseo de control; búsqueda de poder y estatus; obtención de placer; agresividad como parte de su imagen; conciencia de la práctica del acoso. En una nueva síntesis se presentó una crítica reflexiva de los hallazgos revisados. Aunque el número de referencias incluidas en la revisión es limitado, esta metasíntesis es relevante en la medida en que arroja luz sobre las experiencias de los niños que practican el acoso escolar y esta evidencia puede orientar las acciones de intervención contra el acoso.
\end{abstract}

Palabras clave: Violencia; Salud escolar; Acoso; Metasíntesis; Revisión.

\title{
1. Introdução
}

O bullying, modalidade de violência escolar, é um problema de saúde pública que de acordo com o estudo de Marcolino et al. (2018), que utilizou dados da Pesquisa Nacional de Saúde do Escolar (PeNSE) (2012), abrange 7,2\% dos estudantes brasileiros como vítimas e 20,8\% como agressores. Para que um episódio de violência escolar seja classificado como bullying, as seguintes características devem estar presentes: ser um comportamento indesejado e agressivo, que aconteça de forma repetitiva e por um longo período, em uma relação entre pares baseado em um desequilíbrio de poder real ou percebido (Olweus, 2004; Stopbullying, 2020). Esse tipo de violência é considerado como um padrão de comportamentos e não relacionado a eventos únicos (Organização das Nações Unidas para a Educação, a Ciência e a Cultura, 2019). Um grande fator de complicação para o seu reconhecimento é que esse padrão de comportamento acaba acontecendo de forma escondida das autoridades escolares, passando, muitas vezes, despercebido (Costa et al., 2021; Tognetta \& Rosário, 2013) ou, quando não, sendo negligenciado e, de certo modo, normalizado, só recebendo atenção quando as agressões culminam em suicídios, homicídios e/ou ganhando repercussões negativas na mídia (Pigozi \& Machado, 2015). Exemplo disso em território brasileiro foi o episódio conhecido como o "Massacre de Realengo/ Rio de Janeiro" em que uma suposta vítima de bullying, em 2011, invadiu sua antiga escola, matou 12 crianças com tiros de revólver e, então, suicidou-se (Lopes, 2012).

O bullying também pode receber algumas classificações que se diferenciam pela forma com que a agressão é manifestada: a) tipo físico (bater, chutar, uso de armas etc.); b) tipo verbal ou psicológico (ameaças, apelidos, humilhação etc.); e c) tipo indireto (exclusão social, indiferença etc.). Vale acrescentar que nos dias de hoje é possível identificar uma modalidade virtual de sua ocorrência: o cyberbullying - agressões acontecem por meio de dispositivos tecnológicos como o celular ou computador e a questão da repetitividade é relativizada (Marcolino et al., 2018). William e Guerra (2015) ressaltam que as interações sociais já não se restringem aos encontros presenciais (sala de aula), sendo ampliadas para os contextos virtuais (chats).

Sobre a dinâmica do fenômeno, observa-se o desenvolvimento de três papéis desempenhados na dinâmica do fenômeno. Os agressores são os responsáveis pelos comportamentos de violência - os que a praticam. As vítimas são os alvos da violência e os espectadores aqueles que presenciam a violência e podem assumir papel ativo ou passivo diante das agressões testemunhadas. Estudos apontam que todos os envolvidos nesta dinâmica, independentemente do papel ocupado, são impactados negativamente na saúde, desenvolvimento e em termos educacionais (Organização das Nações Unidas para a Educação, a Ciência e a Cultura, 2019). Os dados alarmantes de prevalência e as consequências deletérias do fenômeno para todos os envolvidos reforçam o quão imprescindível são necessários investimentos a níveis científico e político para compreender melhor o tema e, então, elaborar medidas possíveis para minimizar sua ocorrência, levando em conta as suas diversas formas de manifestação e particularidades. Esses argumentos são defendidos por outros estudos (Oliveira et al. 2016; 
Oliveira et al., 2015; Oliveira et al., 2016; Oliveira et al. 2017).

Nesse sentido, no que se refere às diferenças de gênero, segundo a literatura científica, os estudantes do sexo masculino são os mais envolvidos em situações de bullying na escola, principalmente como agressores físicos. O relatório sobre a situação mundial da violência escolar e bullying, publicado em 2017 pela UNESCO (Organização das Nações Unidas para a Educação, a Ciência e a Cultura), cita uma pesquisa realizada em Malta no ano de 2014, pela UNICEF (Fundo das Nações Unidas para a Infância) e que verificou $61 \%$ da amostra dos meninos praticantes do bullying por meio da agressão do tipo físico ante 30\% das meninas. Os estudos de Brito e Oliveira (2013) e de Silva, Dascanio e Valle (2016), apontam que há um maior percentual de vitimização nos diversos tipos de bullying entre escolares do sexo feminino. Em contrapartida, estudos como os de Silva et al. (2013) e Tsitsika et al. (2014) demonstraram que os estudantes de sexo masculinos têm um maior envolvimento nos papéis de vítimas e agressores, principalmente em bullying do tipo físico. Bandeira e Hutz (2012) ainda indicam que muitos meninos participam do bullying não só como agressores, mas também como vítimas-agressoras. Mello et al. (2017) concluíram que além de haver um maior número de agressores do sexo masculino, há uma dominância das seguintes características entre eles: comportamentos que geram risco à saúde (consumo de álcool e drogas, por exemplo) e problemas relacionados a saúde mental (solidão, insônia e não ter amigos, por exemplo).

Esse cenário explicita a necessidade de se particularizar análises a partir do sexo ou gênero dos estudantes envolvidos nas situações de bullying. Será que há diferenças entre meninos e meninas que praticam bullying? Quais as características dos meninos e das meninas identificados como agressores? Essas são perguntas norteadoras do estudo em tela. Para tanto, objetivou-se sintetizar e interpretar estudos primários qualitativos sobre a prática de bullying escolar.

\section{Método}

\subsection{Tipo de estudo}

Trata-se de uma síntese qualitativa desenvolvida a partir da execução de uma revisão sistemática da literatura seguida da integração dos resultados encontrados em estudos qualitativos previamente selecionados. Esse tipo de revisão possui 3 passos: 1) estruturação objetiva (seleção, inclusão e avaliação dos artigos); 2) rigor científico (análise dos dados); e 3) subjetividade do pesquisador (construção da nova síntese), oferecendo percepções originais a partir do corpus revisado (Lachal et al. 2017). Assim, uma síntese qualitativa foi realizada a fim de explorar as características dos agressores de bullying e as diferenças na prática da agressão entre os gêneros, já que essas informações podem ser relevantes para a elaboração de estratégias de intervenção com maior potencial de assertividade e eficiência.

\subsection{Estratégia de busca e questão norteadora}

A estratégia SPIDER (Cooke et al., 2012) foi utilizada para elaboração da seguinte questão norteadora: Quais as evidências científicas qualitativas sobre as experiências de meninos e meninas que praticam bullying na escola? Essa questão contempla os seguintes elementos: Sample (amostra/participantes); Phenonemon of Interest (fenômeno de interesse); Design (desenho do estudo); Evaluation (avaliações/resultados); Research type (tipo de pesquisa) (Cooke et al., 2012). Nesse estudo a amostra deveria ser constituída por estudantes do ensino fundamental ou médio, sendo o bullying escolar o fenômeno de interesse, o desenho dos artigos a serem incluídos deveria ser definido a partir do uso de entrevista ou grupo focal na coleta de dados, sendo evidenciado as experiências dos agressores nos resultados e apenas pesquisas de caráter qualitativo seriam incluídas.

Entre os meses de outubro e novembro de 2020 foram consultadas as seguintes bases de dados: PsycINFO, SCOPUS, Web of Science e SciELO, sendo que em cada uma delas houve uma adaptação na operacionalização da busca, dadas as suas particularidades. Os descritores "bullying", "attacker", "assaulter", "bullie" e "aggressor" foram utilizados em todas as bases 
de dados, exceto no SciELO em que foram utilizados apenas dois descritores ("bullying" e "bullying at school"). Além da busca nas bases de dados mencionadas, foi realizada uma busca no periódico International Journal of Bullying Prevention. Para tanto se utilizou os termos não controlados "qualitative research" e "qualitative study". Essa alternativa se justifica pelo baixo número de estudos qualitativos encontrados nas bases selecionadas e pela relevância do periódico para abordar o fenômeno de interesse da revisão.

\subsection{Critérios de inclusão e exclusão}

Foram incluídos estudos qualitativos primários em português, inglês e espanhol. Não foi estabelecido recorte temporal. Também se incluiu apenas artigos científicos publicados em periódicos. Foram excluídos estudos de revisão, estudos mistos e quantitativos. Também se excluiu estudos com adultos ou sobre bullying em outros contextos que não as escolas.

\subsection{Seleção dos estudos e extração de dados}

Considerando os critérios de inclusão e exclusão e as bases de dados consultadas foram encontrados 574 artigos, excluindo os duplicados se somou 568 artigos. Após a primeira filtragem - leitura dos títulos e resumos - 560 estudos foram retirados. Com a última etapa de filtragem - leitura integral dos textos - dois artigos foram excluídos por não atenderem integralmente aos critérios de inclusão supracitados. Detalhes desse processo estão explicitados no item Resultados e Discussão e no fluxograma PRISMA (Figura 1).

\subsection{Avaliação de qualidade metodológica}

Para a avaliação da qualidade metodológica dos estudos incluídos foram utilizados os critérios do CASP (Critical Appraisal Skills Programme), que é um instrumento delineado para avaliar pesquisas qualitativas (Critical Appraisal Skills Programme, 2018). Este instrumento possibilitou a avaliação dos seguintes itens dos estudos selecionados: declaração clara dos objetivos; uso correto da metodologia qualitativa; desenho de pesquisa apropriado; coleta de dados coerente com a questão de pesquisa; questões éticas; rigor da análise de dados; resultados declarados de forma clara e contribuição original. Essa etapa foi realizada pelo pesquisador responsável pelo estudo (WAO).

\subsection{Análise dos dados}

A análise dos dados consistiu na elaboração de uma síntese qualitativa composta por quatro etapas (Sandelowski \& Leeman, 2012). Na primeira etapa os estudos selecionados foram lidos de forma exaustiva com a intenção de favorecer a reunião de um conjunto sintetizável de informações. A segunda etapa consistiu na codificação dos dados. Em seguida, os códigos foram agrupados e categorizados. Por fim, na quarta etapa, gerou-se temas analíticos. A análise dos resultados foi estruturada em três categorias. A primeira categoria contém os dados bibliométricos, as teorias ou conceitos utilizados (análise metateórica). A segunda categoria (análise metamétodo) incluiu dados dos métodos utilizados na coleta de dados em cada estudo e a avaliação da qualidade metodológica. Por fim, a terceira categoria (metassíntese) reuniu os dados que, efetivamente, respondem ao objetivo proposto na revisão.

\section{Resultados e Discussão}

Após as buscas nas bases de dados foi realizada a leitura dos títulos e resumos dos estudos encontrados. Observa-se que o resultado das buscas com a limitação por tipo de pesquisa não favoreceu retornos numericamente satisfatórios, por isso foram considerados todos os estudos encontrados e o refinamento para identificação dos estudos qualitativos ocorreu durante esse exercício de leitura dos títulos e resumos. Nessa primeira etapa, foram selecionados oito estudos para a leitura integral dos 
textos (considerando os critérios de inclusão e exclusão apresentados). Para detalhar o processo de seleção e resultados foi elaborado um fluxograma segundo as diretrizes PRISMA (Figura 1). O corpus da revisão foi constituído por seis artigos.

Figura 1. Diagrama do fluxo do processo de busca e seleção dos artigos incluídos na revisão (PRISMA).

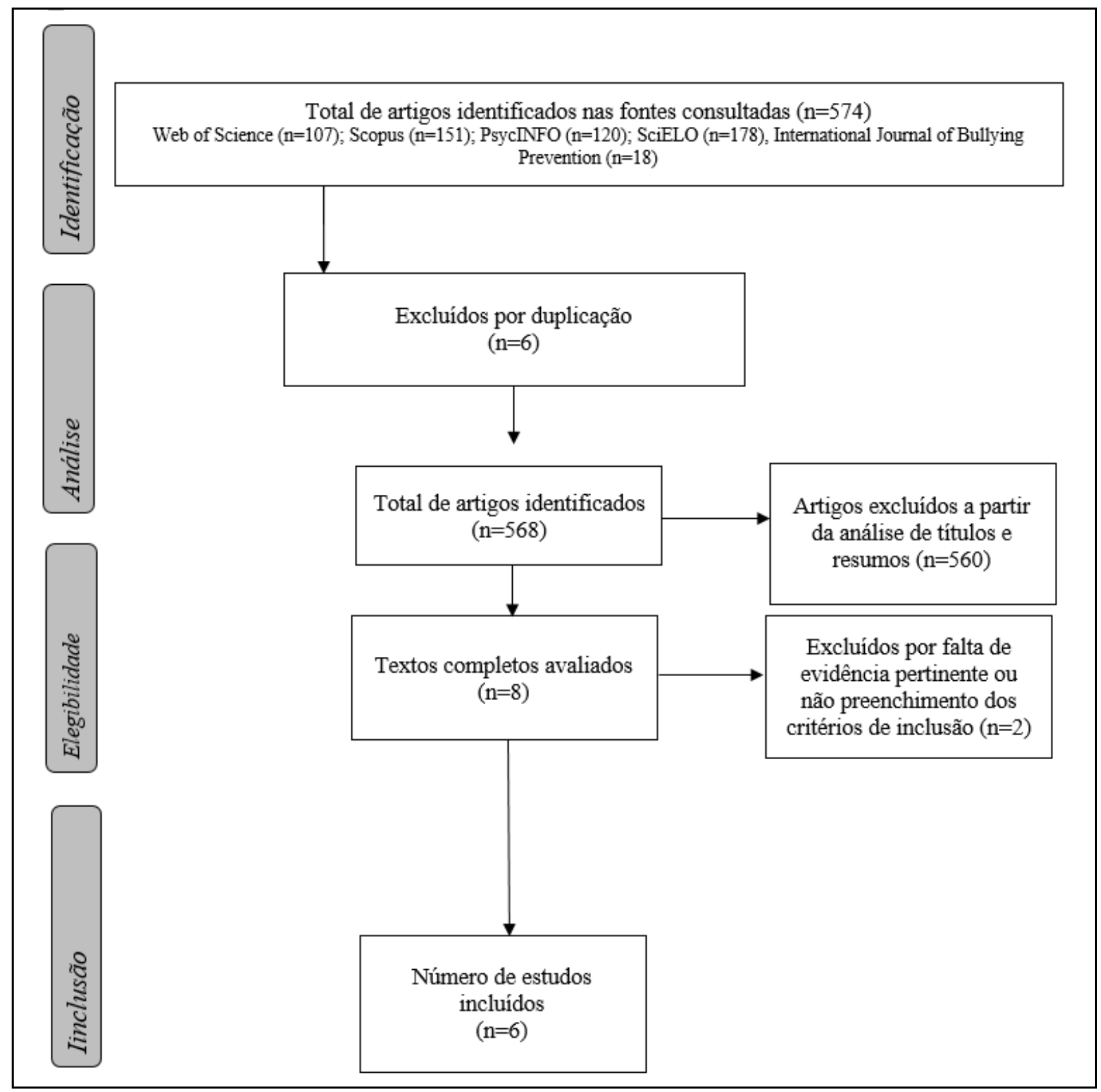

Fonte: Autores.

\subsection{Análise Metatéorica}

Essa categoria busca analisar as características bibliométricas dos artigos que compõem o corpus da revisão. Alguns estudos utilizam as palavras "intimidações" (Nascimento \& Menezes, 2013), "relações de poder" (Forero-Rocha \& GómezRódriguez, 2016), "relações agressivas" (Ródriguez-Machain et al., 2016) e "cultura de agressão" (Myburgh et al., 2015) para se referir ao bullying, enquanto outros utilizam o próprio termo "bullying" para se referir ao fenômeno (Nashiki, 2013; Sainju et al., 2021). Os estudos definem o bullying a partir da conceitualização proposta por Olweus (2004), compreendendo-o como comportamento agressivo e indesejado, repetitivo, com longa duração de tempo e presente em uma relação entre pares com desequilíbrio de poder. No entanto, o estudo de Sainju et al. (2021) utilizou experiências autorrelatadas e interpretações dos próprios autores dos tweets analisados para definir o problema, não podendo garantir as características de poder e repetição que 
estão inseridos na definição do autor supracitado.

Os estudos foram publicados entre 2013 e 2016. Todavia, essa informação não pode ser utilizada para dizer que não houve publicações em outros anos, uma vez que estas produções podem não ter se adequado na aplicação dos critérios de inclusão do presente estudo ou não foram identificadas a partir das estratégias de busca empreendidas. Em relação aos países onde as pesquisas foram realizadas, registrou-se Colômbia $(\mathrm{n}=1)$ (Forero-Rocha \& Gómez-Ródriguez, 2016), México $(\mathrm{n}=2)$ (Nashiki, 2013; Ródriguez-Machain et al., 2016), África do Sul (n=1) (Myburgh et al., 2015), Canadá (n=1) (Sainju et al., 2021) e Brasil (n=1) (Nascimento \& Menezes, 2013). Os autores dos artigos são das áreas da educação ou da saúde. As principais abordagens teóricas adotadas pelos estudos incluídos nessa revisão são de perspectiva social, como a noção de poder de Yulk (Forero-Rocha \& Gómez-Ródriguez, 2016), a noção de identidade de Goffman (Nashiki, 2013), a perspectiva sociocultural da psicologia e do desenvolvimento humano (Nascimento \& Menezes, 2013).

Os artigos (Forero-Rocha \& Gómez-Ródriguez, 2016; Nashiki, 2013; Ródriguez-Machain et al., 2016; Myburgh et al., 2015; Nascimento \& Menezes, 2013) relacionam, majoritariamente, as agressões diretas de ordem física (como chutes e empurrões) em sobreposição às agressões indiretas (como fofocas e atitudes de exclusão) para os meninos e o inverso (agressão indireta em sobreposição à agressão direta) para as meninas. Essa diferença será mais amplamente explorada na terceira categoria de análise do presente estudo, entretanto cabe aqui ressaltar que esta informação não significa a ausência de atitudes de exclusão entre meninos e de agressões físicas entre meninas, sendo apenas utilizadas em termos de menor ou maior frequência.

Em relação aos objetivos, o estudo de Nascimento e Menezes (2013) pretendeu compreender os significados produzidos sobre as práticas de intimidação em contexto escolar, enquanto o de Forero-Rocha, Gómez-Ródriguez (2016) observar em profundidade como a desigualdade de poder foi estabelecida por um grupo de jovens alunos de inglês em uma escola pública. Já a pesquisa de Ródriguez-Machain et al. (2016) objetivava conhecer a forma como os estudantes do ensino médio da Cidade do México se relacionam e o de Myburgh et al. (2015) relatar os padrões de uma cultura de agressão observada entre os alunos da décima série em uma escola no distrito de Sedinbeng (África do Sul). Nashiki (2013) objetivou analisar como atuam os alunos agressores e os argumentos que manifestam, além de analisar a forma em que as vítimas vivem. Por fim, Sainju et al. (2021) objetivaram analisar as descrições de episódios de bullying offline e online fornecidos em tweets e descrever as características relacionadas com o agressor, o alvo e o ajudante. Por meio da análise metateórica e dos objetivos citados de cada estudo, compreende-se que existe um interesse científico em ampliar o conhecimento acerca da experiência da prática do bullying escolar a partir dos agressores e por meio de pesquisas qualitativas.

\subsection{Análise metamétodo}

Verificou-se que os pesquisadores utilizaram diferentes técnicas para coleta de dados. A técnica de coleta de dados predominante foi a observação. Notas de campo apoiadas em gravações, questionários, diários, por exemplo, em um estudo que contou com 36 participantes, sendo 23 meninas e 13 meninos com idades entre 15 e 19 anos (Forero-Rocha, GómezRódriguez, 2016). Observação do contexto foi aplicada em três estudos com as seguintes características: 28 estudantes entre 16 e 18 anos (Nascimento \& Menezes, 2013); 15 estudantes, sete pais, oito integrantes da equipe de gestão escolar e oito integrantes do corpo diretivo de uma escola (Myburgh et al., 2015); e 40 estudantes de 13 a 15 anos do segundo e terceiros anos do ensino médio, sendo 10 meninas e 10 meninos com condutas agressivas anteriores e 10 meninas e 10 meninos sem registro de tais condutas (Ródriguez-Machain et al., 2016). Grupos focais (Nascimento \& Menezes, 2013; Ródriguez-Machain et al., 2016), entrevistas e notas de campo (Myburgh et al., 2015) foram outras técnicas aplicadas. A coleta de dados em mídias sociais foi aplicada em um estudo, sendo coletados tweets entre 07 de agosto de 2019 e 31 de março de 2020 por meio das palavras-chave "bullied", "bully", "bullying", "cyberbullies", "cyberbully" e "cyberbullying", sendo inclusos 240.018 tweets 
(Sainju et al., 2021). Dois estudos (Nascimento \& Menezes, 2013; Myburgh et al., 2015) que não revelaram a quantidade de meninos e meninas na amostra fizeram especificações do sexo no momento de utilizar dados como falas ou comportamentos considerados nos resultados.

Os estudos revisados não fizeram uso de softwares para realizar a análise dos dados. Foram explicitadas as seguintes estratégias ou abordagens de análise: abordagem fundamentada, organização temática da informação, abordagem de Tesch, núcleo de significações de Ozella e categorização. Nem todos os artigos mencionaram as questões éticas.

A análise da qualidade metodológica, que utilizou os critérios do CASP (Critical Appraisal Skills Programme, 2018), pode ser observada na Tabela 1.

Pode-se perceber que a pontuação da qualidade dos métodos dos artigos ficou entre 8 e 9 pontos, com exceção de um artigo que totalizou 6 pontos (Nashiki, 2013). Este artigo teve uma baixa pontuação por não ter realizado uma estratégia de coleta de dados coerente com os objetivos, não ter considerado corretamente a relação pesquisador-participante, não ter considerado as questões éticas e coleta de dados insuficientemente rigorosas. No entanto, os outros artigos foram bem classificados e, portanto, possuíam um baixo risco de viés e com alto ou moderado poder de evidência.

\subsection{Metassíntese}

Em relação às características dos agressores, verificaram-se um total de seis nos estudos analisados: alto senso de superioridade (Forero-Rocha \& Gómez-Ródriguez, 2016), desejo de controle (Nascimento et al., 2013; Forero-Rocha \& Gómez-Ródriguez, 2016), busca de poder e status (Nashiki, 2013; Nascimento et al., 2013; Ródriguez-Machain et al., 2016; Forero-Rocha \& Gómez-Ródriguez, 2016), obtenção de prazer com a irritação alheia (Forero-Rocha \& Gómez-Ródriguez, 2016), agressividade como constituinte de sua imagem (Ródriguez-Machain et al., 2016) e consciência da prática da intimidação (Sainju et al., 2021). Um dos estudos trouxe a informação de que os agressores manifestaram remorso por ter praticado bullying (Sainju et al., 2021).

Dados quantitativos do estudo de Silva et al. (2019) apontam que, embora ambos os sexos possam ser agressores, este papel é ocupado principalmente por meninos. Os artigos analisados no presente estudo concordam com este achado. A naturalização da violência nas relações entre pares está presente tanto em meninos quanto em meninas, mas o tipo de violência predominante muda conforme o sexo. Os primeiros praticam a violência direta (física) enquanto as meninas praticam a violência indireta (psicológica e verbal), majoritariamente (Nashiki, 2013; Forero-Rocha \& Gómez-Ródriguez, 2016). Isto pode ser exemplificado com a fala de um dos integrantes da equipe de gestão escolar que participou do estudo de Nashiki (2013): "Os meninos são muito mais físicos e as meninas usam a boca". Considerar a particularidade da prática de agressão para cada sexo pode propiciar maior assertividade em programas de intervenção. 
Research, Society and Development, v. 11, n. 2, e18111225668, 2022

(CC BY 4.0) | ISSN 2525-3409 | DOI: http://dx.doi.org/10.33448/rsd-v11i2.25668

Tabela 1. Resultados da avaliação de qualidade metodológica do corpus revisado.

\begin{tabular}{|c|c|c|c|c|c|c|c|c|c|c|c|}
\hline Referência & $\begin{array}{l}\text { Houve uma } \\
\text { declaração } \\
\text { clara dos } \\
\text { objetivos da } \\
\text { pesquisa? }\end{array}$ & $\begin{array}{l}\text { Usa } \\
\text { metodologia } \\
\text { qualitativa } \\
\text { apropriada? }\end{array}$ & $\begin{array}{l}\text { O desenho da } \\
\text { pesquisa foi } \\
\text { apropriado } \\
\text { para abordar } \\
\text { seus } \\
\text { objetivos? }\end{array}$ & $\begin{array}{l}\text { A estratégia } \\
\text { de } \\
\text { recrutamento } \\
\text { foi adequada } \\
\text { aos objetivos } \\
\text { da pesquisa? }\end{array}$ & $\begin{array}{l}\text { Os dados } \\
\text { foram } \\
\text { coletados de } \\
\text { forma a } \\
\text { abordar a } \\
\text { questão da } \\
\text { pesquisa? }\end{array}$ & $\begin{array}{l}\text { A relação } \\
\text { entre o } \\
\text { pesquisador e } \\
\text { os } \\
\text { participantes } \\
\text { foi } \\
\text { considerada } \\
\text { de forma } \\
\text { adequada? }\end{array}$ & $\begin{array}{l}\text { As questões } \\
\text { éticas foram } \\
\text { levadas em } \\
\text { consideração? }\end{array}$ & $\begin{array}{l}\text { A análise dos } \\
\text { dados foi } \\
\text { suficientemen } \\
\text { te rigorosa? }\end{array}$ & $\begin{array}{l}\text { Existe uma } \\
\text { declaração } \\
\text { clara das } \\
\text { descobertas/ } \\
\text { resultados? }\end{array}$ & $\begin{array}{l}\text { Quão valiosa } \\
\text { é a pesquisa } \\
\text { ou qual a sua } \\
\text { contribuição } \\
\text { original? }\end{array}$ & Total \\
\hline $\begin{array}{l}\text { Forero-Rocha \& Gómez- } \\
\text { Rodríguez, } 2016\end{array}$ & S & $\mathrm{S}$ & S & S & S & $\mathrm{N}$ & $\mathrm{N}$ & S & S & S & 8 \\
\hline Myburgh et al., 2015 & $\mathrm{~S}$ & $\mathrm{~S}$ & $\mathrm{~S}$ & $\mathrm{~S}$ & $\mathrm{~S}$ & $\mathrm{~N}$ & $\mathrm{~S}$ & $\mathrm{~S}$ & $\mathrm{~S}$ & $\mathrm{~S}$ & 9 \\
\hline $\begin{array}{l}\text { Nascimento \& Menezes, } \\
2013\end{array}$ & $\mathrm{~S}$ & $\mathrm{~S}$ & $\mathrm{~S}$ & $\mathrm{~S}$ & $\mathrm{~S}$ & $\mathrm{~N}$ & $\mathrm{~S}$ & $\mathrm{~S}$ & $\mathrm{~S}$ & $\mathrm{~S}$ & 9 \\
\hline Nashiki, 2013 & S & $\mathrm{S}$ & S & $\mathrm{N}$ & S & $\mathrm{N}$ & $\mathrm{N}$ & $\mathrm{N}$ & $\mathrm{S}$ & $\mathrm{S}$ & 6 \\
\hline $\begin{array}{l}\text { Rodríguez-Machain et al., } \\
2016\end{array}$ & S & S & S & $\mathrm{NI}$ & S & $\mathrm{N}$ & S & S & S & S & 8 \\
\hline Sainju et al., 2021 & $\mathrm{~S}$ & $\mathrm{~S}$ & $\mathrm{~S}$ & $\mathrm{~S}$ & $\mathrm{~S}$ & $\mathrm{~N}$ & $\mathrm{~S}$ & $\mathrm{~S}$ & $\mathrm{~S}$ & $\mathrm{~S}$ & 9 \\
\hline
\end{tabular}

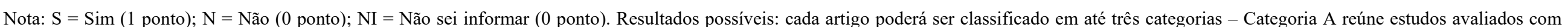

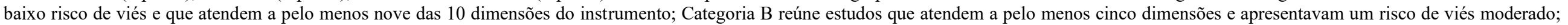
Categoria $\mathrm{C}$ reúne estudos que atendem quatro ou menos dimensões e apresentavam alto risco de viés. Fonte: Autores. 
De acordo com o relato dos meninos apresentados nos estudos, tanto a violência direta quanto a indireta fazem parte do vínculo entre pares (Ródriguez-Machain et al, 2016), pensamento que é reforçado pela cultura da agressividade masculina. Nesta linha, a demarcação da identidade dos adolescentes meninos acaba por ser alcançada através da distinção entre o enturmado (detentor de status) e excluído (vítima de bullying, por exemplo). Observa-se, então, uma contínua afirmação e reafirmação das relações de poder (Silva, 2011). Morgante (2014) afirma que um problema reconhecido é a presença da agressividade como um componente da identidade masculina, o que vai ao encontro às expectativas desta população de necessidade de detenção do domínio, razão e poder nas relações. Interessante observar essa relação entre agressividade e masculinidade inclusive na fala de uma menina vítima de agressões indiretas realizadas por outras meninas: "São umas 'marimachas', disse minha mãe, e por isso não se dedicam a nada mais além de se divertirem com meu cabelo, minha roupa e até minha casa, porque já foram me irritar até em minha casa, me gritaram e disseram coisas muito grosseiras". (Nashiki, 2013). A expressão "marimachas" é usada para ressaltar atitudes e estereótipos de meninos na agressão realizada por meninas.

Sobre a característica "necessidade de controle sobre o outro", dois artigos revisados citam os seguintes recursos utilizados para atingir tal meta: ameaças, denotações recorrentes e diversas formas de intimidação (Nashiki, 2013; ForeroRocha \& Gómez-Ródriguez, 2016; Nascimento et al., 2013). Um questionamento parte dessa informação com base na literatura científica sobre a violência doméstica, segundo a qual esta mesma característica está presente no padrão de comportamento do agressor em relação a vítima (Melatti, 2015). Partindo da premissa de certo padrão comportamental, inferese que há a possibilidade de estudantes agressores se tornarem futuros parceiros agressores. Em caso de confirmação dessa inferência, salienta-se a importância da intervenção em comportamentos de bullying como sendo uma possível medida protetiva para a violência em relações afetivas nos outros momentos do ciclo vital.

O estudo de Nashiki (2013) possibilita também exemplificar o desejo de obtenção de poder e status por meio do bullying através da fala de um dos integrantes da amostra. $\mathrm{O}$ autor observou essa função dos comportamentos agressivos através de falas de alguns alunos participantes da amostra de sua pesquisa, sendo sete do sexo masculino e quatro do sexo feminino, sobre o bullying. Segundo eles, eleger uma vítima faz com que possam mostrar o seu poder e controle. Inclusive, os agressores parecem apresentar certa obsessão para encontrar mais vítimas, pois elas serviriam como "colecionar troféus de guerra". O estudo de Forero-Rocha e Gómez-Ródriguez (2016), em específico, relata que as observações realizadas durante uma aula de inglês de contexto escolar indicaram a criação de um ambiente inseguro para as vítimas e as agressões apareciam em forma de risos decorrentes dos erros cometidos devido a falta de conhecimento da língua estrangeira (pronúncia, gramática etc.). Este é um outro exemplo da característica de "desejo de obtenção de poder e status" (quem sabe e quem sabe a língua em estudo), também retratadas pelo estudo de Nascimento et al. (2013). Em consonância, Nashiki (2013) afirma que a agressão acaba sendo uma espécie de tentação, uma vez que é entendida como uma forma de exercer o poder.

O estudo de Rodriguez-Machain et al. (2016) aborda a presença da violência direta e indireta na constituição do vínculo entre pares masculinos e femininos. Porém, na perspectiva masculina este comportamento faz parte, inclusive, dos vínculos de amizade, enquanto na perspectiva feminina é o vínculo existente entre as envolvidas que irá diferenciar se estas mesmas atitudes serão consideradas como parte integrante da amizade ou como agressão. Também é abordada a dificuldade por parte dos alunos meninos constituinte da amostra em rotular alguém como "agressor" ou "vítima", uma vez que estes papéis eram comumente intercalados entre os integrantes, ou sejam, um mesmo indivíduo ser ora agressor ora alvo. O trabalho apresenta a questão da cultura de violência entre meninos como sendo amplamente aceita e naturalizada, como já dito anteriormente, ao passo que se espera das mulheres comportamentos mais dóceis ou opostos à agressividade. Assim, o debate sobre este aspecto deve constituir o alicerce para o desenvolvimento de intervenções com agressores meninos, destacando nessa abordagem tanto a presença da violência nas relação menino-menino quanto menino-menina.

Além disso, a partir do estudo de Nashiki (2013), que utilizou entrevistas, diários de campo e observações para a 
análise da atuação dos agressores e dos argumentos utilizados para tal, foi possível demonstrar a presença da desvalorização do feminino por meio do uso de termos femininos durante a agressão verbal entre meninos. O estudo de Forero-Rocha e GómezRódriguez (2016) também demonstrou tal desvalorização, desta vez como argumentação para a ridicularização das meninas por parte dos meninos, uma vez que estas "não eram dignas de serem vistas ou ouvidas" e não deveriam fazer parte de nenhum grupo. Também foram relatadas falas como "Gabriel e David ofendem as meninas com comentários maldosos (...)".

Pode-se destacar a violência de gênero demonstrada por estes estudos como sendo a materialização da necessidade de detenção da dominância cuja raiz se encontra no imaginário acerca da agressividade como parte integrante da identidade masculina. Tal ideia se fortalece a partir de uma cultura que relaciona passividade e afetividade (características que costumam ser associadas ao feminino) como sinais de inferioridade, como características opostas à masculinidade (Morgante, 2014). Esse aspecto é também experimentado nas situações de bullying, como explicitado nos estudos revisados.

Sobre a forma como a desigualdade de poder, característica do bullying, é criada e mantida durante a aula de inglês de uma escola pública em Bogotá observada no estudo de Forero-Rocha e Gómez-Rodríguez (2016) a respeito das relações de poder durante a disciplina, verificou-se que os agressores (todos de sexo masculino) e seus companheiros lançam mão de recursos como a interrupção indelicada quando a vítima está participando da aula. Os autores ressaltam que tal atitude não pode ser enxergada apenas como uma questão de indisciplina, já que esses alunos, além de interromperem de forma abrupta a vítima, também corrigiam a pronúncia dos estudantes que estavam respondendo à pergunta realizada pelo professor com um tom de zombaria e hostilidade, e concluem que esses comportamentos tinham como função fazer com que se tornassem o centro das atenções.

O estudo de Sainju et al. (2021) retrata a consciência da prática de intimidação através dos registros encontrados nos tweets analisados. Em consonância, Nashiki (2013) traz a seguinte fala de um agressor do sexo masculino: "eu os violento porque (...) me aborreço, porque sim (...), me aborreço e assim me distraio e aqui todos já sabemos como se faz, já sabem que assim eu me relaciono com eles, não vou andar os abraçando (...)". Percebe-se que tal problemática se torna ainda mais relevante, já que, para intervir eficazmente em situações como esta, é necessário identificar o fenômeno. Este dado é abordado na literatura científica, que confirma o despreparo dos profissionais na identificação do bullying (Silva et al., 2014). Embora este comportamento ocorra principalmente em ambiente escolar, é difícil para a maioria dos professores notá-los em toda a sua dimensão/complexidade.

\subsection{Nova síntese}

Partindo dos dados encontrados e supracitados, reflete-se que o sofrimento causado pela dinâmica do bullying não atinge só as vítimas, mas também os agressores. O bullying deve ser entendido como um fenômeno complexo e multidimensional não só em sua origem, mas também em suas repercussões. Para além dos problemas educacionais, desenvolvimentais e de saúde propagados pela literatura científica, faz-se necessário pensar sobre o sofrimento emocional que parte da necessidade não só da obtenção do poder e status a qualquer custo, mas também de sua manutenção. $\mathrm{O}$ agressor se vê precisando, de todas as formas e a todo o momento, esconder vulnerabilidades características do ser humano. Perde a humanidade não só ao adotar uma postura de invulnerabilidade e bem-estar contínuos, mas também ao não reconhecer a humanidade do outro, sendo capaz de agredir física e verbalmente seus pares. Ocorre uma invalidação mútua: invalida o outro ao mesmo tempo que se invalida, escondendo tal ciclo por trás de uma imagem irreal de si mesmo e de uma busca de validação externa.

Não se pretende com o exposto acima justificar as atitudes de agressão ao se enxergar o bullying como um ato de violência contra si mesmo sendo manifestado através de uma violência aos outros. Se pretende propor um olhar cuidadoso com estes alunos, que causam sofrimento alheio e pessoal de forma concomitante. Partindo disso, uma outra estratégia para intervir 
no bullying escolar pode partir de atividades que proponham o acolhimento das próprias vulnerabilidades dos alunos, do reconhecimento de si e do outro como seres humanos, de forma a abrir espaço para a desnaturalização da violência e a naturalização do "ser" humano. Esse tipo de estratégia parece ser ainda mais válido para o caso dos meninos.

Além disso, os estudantes devem receber, no contexto educacional, ensinamentos para além das disciplinas acadêmicas tradicionais. A escola deve ser um ambiente que propicie o ensinamento do respeito à dignidade humana, onde haja o desenvolvimento de relações saudáveis que influenciarão nas relações futuras dos estudantes. É contraditório pensar que o contexto que deveria oferecer segurança e sensação de pertencimento aos meninos e meninas acaba sendo, para muitos, sinônimo de insegurança e solidão - como evidenciado nos estudos revisados.

Não é tarefa fácil tomar como objeto de estudo atitudes de agressão. O estudo da metodologia qualitativa aumenta ainda mais essa dificuldade ao colocar o pesquisador em contato com falas de sofrimento das vítimas e de certa negligência para com parte dos agressores. A sensação, em um primeiro momento é de afastamento e certa repulsa, mas com o avançar e a imersão na vivência dos agressores é possível minimizar o estigma de "monstruosidade" e reconhecê-los enquanto humanos. Então, tomando o ser humano como ser de potencialidades, é possível acreditar no potencial de mudanças desses alunos, ainda mais ao se considerar a adolescência como um processo de formação da identidade e para o desenvolvimento de diferentes caraterísticas.

\section{Considerações Finais}

Essa revisão resume informações de estudos primários qualitativos sobre a prática de bullying escolar que apresentavam algumas diferenças de gênero. Nesse sentido, evidenciou-se a busca por status e a noção de superioridade por parte dos agressores. O bullying foi apresentado, portanto, como um meio para se atingir tal objetivo e colocar em prática essa desigualdade de poder entre os pares. Os artigos permitiram destaque à diferença de tipo de agressão entre meninos e meninas, sendo a de tipo físico (direto) mais praticado pelos meninos e de exclusão e fofocas (indireto) pelas meninas, e como característica de semelhança encontra-se uma certa naturalização da violência entre pares para ambos os sexos.

Seguindo o método particular escolhido para esta revisão (síntese qualitativa), apenas artigos de pesquisa primária que usaram métodos qualitativos foram incluídos. Assim sendo, reconhece-se que as evidências identificadas foram restritas e devem ser interpretadas considerando o pequeno número de produções identificadas e revisadas. Sobre a avaliação da qualidade metodológica apresentada nessa revisão, salienta-se que não existe um padrão ouro na avaliação de estudos qualitativos e mesmo estudos com algum risco de viés podem fornecer informações valiosas sobre a experiência focalizada (no caso dessa revisão o bullying escolar). Outra limitação a ser mencionada se refere ao fato de não ser objetivo dos estudos revisados verificar diferenças específicas entre meninos e meninas na prática do bullying. Dessa forma, para mitigar essas limitações, recomenda-se novas revisões de literatura que contemplem estudos quantitativos ou mistos e que podem complementar os achados aqui revelados.

Ressalta-se que a produção de mais pesquisas qualitativas sobre o fenômeno é importante para o aprofundamento nas experiências da prática desta modalidade de violência e podem, inclusive, se articular às pesquisas quantitativas, uma vez que estas traduzem numericamente a importância e extensão do problema, já considerado de saúde pública. Como se verificou nessa revisão são poucos os estudos de natureza qualitativa e por isso eles são recomendados.

\section{Referências}

Bandeira, C. M. \& Hutz, C. S. (2012). Bullying: prevalência, implicações e diferenças entre os gêneros. Revista Semestral da Associação Brasileira de Psicologia Escolar e Educacional, 16(1), 35-44. https://doi.org/10.1590/S1413-85572012000100004

Brito, C. C. \& Oliveira, M. T. (2013). Bullying e autoestima em adolescentes de escolas públicas. Jornal de Pediatria, 89(6), 601-607. https://doi.org/10.1016/j.jped.2013.04.001. 
Critical Appraisal Skills Programme. (2018). CASP Qualitative of checklist. https://casp-uk.b-cdn.net/wp-content/uploads/2018/03/CASP-QualitativeChecklist-2018_fillable_form.pdf

Cooke, A., Smith, D., \& Booth, A. (2012). Beyond PICO: the SPIDER tool for qualitative evidence synthesis. Qualitative Health Research, 22(10), 14351143. https://doi.org/10.1177/1049732312452938

Costa, F. G., Coutinho, M. P. L., Cavalcanti, J. G., Coutinho, M. L., \& Fonseca, A. A. R. (2021). Bullying, depression and social representations in the school context. Research, Society and Development, 10(16), e369101623617. https://doi.org/10.33448/rsd-v10i16.23617

Forero-Rocha, Y. \& Gómez-Rodríguez, L. F. (2016). The influence of power relations on teenagers learning process in EFL Class. Revista Latinoamericana de Ciencias Sociales, Niñez y Juventud, 14(2), p.1493-1506. http://dx.doi.org/10.11600/1692715x.14241040915

Lachal, J., Revah-Levy, A., Orri, M., \& Moro, M. R. (2017). Metasynthesis: An Original Method to Synthesize Qualitative Literature in Psychiatry. Frontiers in psychiatry, 8 (269),1-9. https://doi.org/10.3389/fpsyt.2017.00269

Lopes, A. J. (2012). Considerações sobre o Massacre de Realengo. Estudos de Psicanálise, (37), 25-44. http://pepsic.bvsalud.org/scielo.php?script=sci_arttext\&pid=S0100-34372012000100003\&lng=pt\&tlng=pt

Marcolino, E. C., Cavalcanti, A. L., Padilha. W. W. N; Miranda, F. A. N.\& Clementino, F. S. (2018). Bullying: prevalências e fatores associados à vitimização e à agressão no cotidiano escolar. Texto \& Contexto Enfermagem, 27(1), 1-10. https://doi.org/10.1590/0104-07072018005500016

Melatti, K. R. (2015). O bater como ato ideológico: violência doméstica e suas interfaces com a lógica da dominação. Temporalis, 15(29), 289-309. https://doi.org/10.22422/2238-1856.2015v15n29p289-309

Mello, F. C. M., Silva, J. L.; Oliveira, W. O., Prado, R. R., Malta, D. C. \& Silva M. A.I. (2017). A prática de bullying entre escolares brasileiros e fatores associados, Pesquisa Nacional de Saúde do Escolar 2011. Ciências \& Saúde Coletiva, 22(9), 2939-2948. https://doi.org/10.1590/1413-81232017229.12762017

Morgante, M. M. (2014). Identidade masculina e agressividade: limites para o enfrentamento da violência de gênero. Revista Ars Histórica, 9, 138-153. https://historia.ufrj.br/ ars/

Myburgh, C., Poggenpoel, M. \& Nhlapo, L. (2015). Patterns of a culture of aggression amongst grade 10 learners in a secondary school in the Sedibeng District, South Africa. Curationis, 38(1), 1-8. http://dx.doi.org/10.4102/curations.v38I1.1233

Nascimento, A. M. T. \& Menezes, J. A. (2013). Intimidações na adolescência: expressões da violência entre pares na cultura escolar. Psicologia \& Sociedade, 25, 142-151. https://doi.org/10.1590/S0102-71822013000100016

Nashiki, A. G. (2013). Bullying: el poder de la violencia. Revista Mexicana de Investigación Educativa, Mexico, 18(58), 839-870. https://doi.org/10.15174/au.2016.955

Oliveira, W. A., Silva, J. L., Querino, R. A., \& Silva, M. A. I. (2016). Experiences and perceptions of discrimination related to bullying among Brazilian students. Maltrattamento e Abuso all 'infanzia, 18(1), 29-57. https://doi.org/10.3280/MAL2016-001003

Oliveira, W. A., Silva, M. A., Mello, F. C., Porto, D. L., Yoshinaga, A. C., \& Malta, D. C. (2015). The causes of bullying: results from the National Survey of School Health (PeNSE). Revista Latino-Americana de Enfermagem, 23(2), 275-282. https://doi.org/10.1590/0104-1169.0022.2552

Oliveira, W. A., Silva, M. A., Silva, J. L., Mello, F. C., Prado, R. R., \& Malta, D. C. (2016). Associations between the practice of bullying and individual and contextual variables from the aggressors' perspective. Jornal de Pediatria, 92(1), 32-39. https://doi.org/10.1016/j.jped.2015.04.003

Oliveira, W. A., Silva, J. L., Sampaio, J. M. C., \& Silva, M. A. I. (2017). Saúde do escolar: uma revisão integrativa sobre família e bullying. Ciência \& Saúde Coletiva, 22, 1553-1564. https://doi.org/10.1590/1413-81232017225.09802015

Olweus, D. (2004). Conductas de acaso y amenaza entre escolares. Ediciones Morata.

Organização das Nações Unidas para a Educação, a Ciência e a Cultura. (2019). Violência escolar e bullying: relatório sobre a situação mundial. https://prceu.usp.br/repositorio/violencia-escolar-e-bullying-relatorio-sobre-a-situacao-mundial/

Pigozi, P. L. \& Machado, A. L. (2015). Bullying na adolescência: visão panorâmica do Brasil. Ciências \& Saúde Coletiva, 20(11), 3509-3522. http://dx.doi.org/10.1590/1413-812320152011.05292014

Rodriguez-Machain, A. C., Berenzon-Gorn, S., Juárez-Garcia, F., \& Valadez-Figueroa, I. (2016). "Así nos llevamos": um estudio cualitativo sobre las relaciones agresivas entre estudiantes de uma secundaria de la Ciudad de Mexico. Acta Universitaria, 26(3), 77-86. https://doi.org/10.15174/au.2016.955

Sandelowski M, Leeman J. (2012). Writing usable qualitative health research findings. Qualitative Health Research, 22, 1404-13. https://doi.org/10.1177/1049732312450368

Sainju, K. D. Mishra, N. Kuffour, A. \& Young, L. (2021). Bullying-Related Tweets: a qualitative examination of perpretators, targets, and helpers. International Journal of Bullying Prevention, 120. https://doi.org/10.1016/j.chb.2021.106735

Silva, F., Dascanio, D., \& Valle, T. G. M. (2016). O fenômeno bullying: diferenças entre meninos e meninas. Reflexão e Ação, 24(1), 26-46. https://doi.org/10.17058/rea.v24i1.7014

Silva, J. L., Oliveira, W. A., Bazon, M. R. \& Cecilio, S. (2014). Bullying: conhecimentos, atitudes e crenças de professores. Psico, Porto Alegre, 45(2), 147156. https://doi.org/10.15448/1980-8623.2014.2.12683

Silva, J. L., Oliveira, W. A., Mello, F. C. M., Prado, R. R., Silva, M. A. I. \& Malta, D. C. (2019). Prevalência da prática de bullying referida por estudantes brasileiros: dados da Pesquisa Nacional de Saúde do Escolar, 2015. Epidemiologia em Serviços de Saúde, 28(2), 1-11. https://doi.org/10.5123/S167949742019000200005 
Research, Society and Development, v. 11, n. 2, e18111225668, 2022

(CC BY 4.0) | ISSN 2525-3409 | DOI: http://dx.doi.org/10.33448/rsd-v11i2.25668

Silva, M. A. I., Pereira, B., Mendonca, D., Nunes, B., \& Oliveira, W. A. (2013). The involvement of girls and boys with bullying: an analysis of gender differences. International journal of environmental research and public health, 10(12), 6820-6831. https://doi.org/10.3390/ijerph10126820

Silva, T. T. (2011). Identidade e diferença: a perspectiva dos estados culturais. (10a ed.), Vozes.

Stopbullying. (2021, 17 janeiro). What is bullying. http://www.stopbullying.gov

Tognetta, L. R. P. \& Rosário, P. (2013). Bullying: dimensões psicológicas no desenvolvimento moral. Estudos em Avaliação Educacional, 24(56), 106-137. http://hdl.handle.net/11449/125165

Tsitsika, A. K., Barlou, E., Andrie, E., Dimitropoulou, C., Tzavela, E. C., Janikian, M., \& Tsolia, M. (2014). Bullying behaviors in children and adolescents: "an ongoing story". Frontiers in public health, 2, 7. https://doi.org/10.3389/fpubh.2014.00007

Williams, K. R. \& Guerra, N. G. (2007). Prevalence and predictor of internet bullying. Journal Adolescent Health, 41(6), 14-21. https://pubmed.ncbi.nlm.nih.gov/18047941/ 